\title{
Production Resource Molding of Grid-based Steel-Auto Industry Chain
}

\author{
Zong-hui Xiong ${ }^{1,2}$, Feng Xiong ${ }^{1}$, Zhen $\mathrm{Li}^{1}$ and Tao $\mathrm{Yu}^{1}$ \\ ${ }^{1}$ Dept. of Mechanical Engineering, Xinjiang University, Urumqi 830008, China \\ ${ }^{2}$ School of Mechatronics Engineering and Automation, Shanghai University, Shanghai 200072, China \\ xiongzonghui@163.com
}

\begin{abstract}
This paper divides steel-auto industry chain from core business and value-added into steel unit, trade unit, machining unit, auto unit and general service unit in the light of the industry chain characteristics. Then product resource in the steel-auto industry chain is defined. By means of Grid technology outstanding advantages in resource sharing, system flexibility and agility, this paper emphasizely studies all information model, formal specification and application Grid-based of product resource.

Key Words-Grid; Supply chain management; Production resource molding; Steel-Auto Industry Chain
\end{abstract}

\section{Introduction}

As the rising important information technology in the international in recent years, research goal of grid technology is to change the low utilization of Internet resources in the current situation, and integrate all available resources $n$ the network together effectively and eliminate isolated information Island and island resources to implement resource sharing and collaboration work in multiple virtual community [1-2]. The information of grid-based supply chain information system can be more easily across organizational boundaries in the supply chain so as to transfer and share among the members. Grid technology has more application space in improving the reliability of the supply chain, weakening the bullwhip effect, improving supply chain flexibility [3].

In the steel-auto industry chain, the main products are steel coil, rolled plate and part. The large amount of information is involved in the product with a wide range, and the products distribute in different countries and organizations, which are very consistent with the dynamic, heterogeneous, distributed and autonomous nature of grid technology. Therefore, it is vital to model and describe product resources in the steel-auto industry chain through grid technology, so as to realize the source collaboration and sharing of the steel-auto industry chain and improve the efficiency of business operations in the overall industrial chain [3].

\section{Product Resource Molding of Steel-Auto Industry Chain}

In the context of economic globalization and information technology, collaboration of steel-auto industry chain is created as a new mode of business operation with the uncertain environment of demand, competition and development. The mode of operation will make the vision of strategic planning chain extended to the entire industry, such as development, design in the upstream, manufacturing and processing in the midstream, and marketing, distribution and services in the downstream. The whole industry chain involves steel manufacturing, processing and distribution companies, third party logistics enterprises, trading companies, auto companies in the downstream and banks, CA authentication center, and other service enterprises, shown in Fig.1.

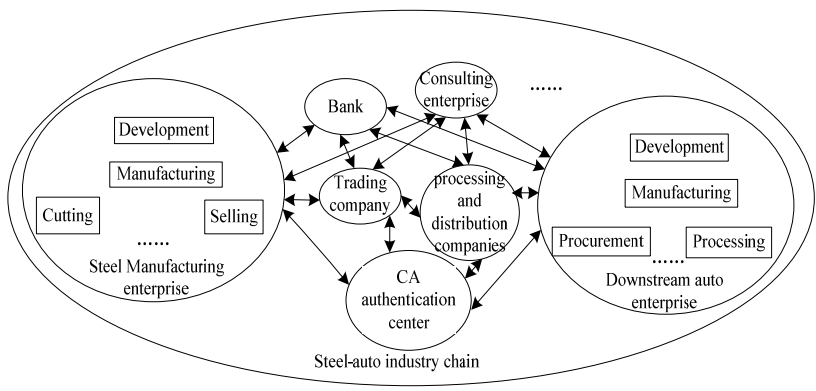

Fig. 1 Enterprises in the steel-auto industry chain

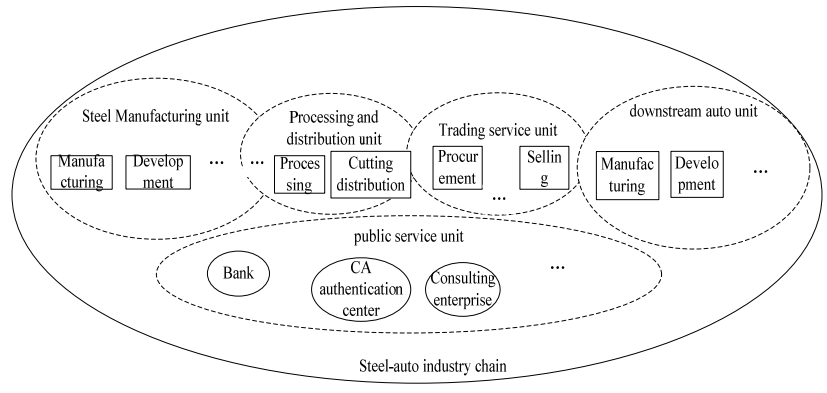

Fig. 2 Units in the steel-auto industry chain

From the perspective of value added and core business, the paper redesigns the enterprises with same or similar value activities or process grouped into one unit, shown in Fig. 2. The whole industry chain is divided into steel manufacturing unit, trading services unit, processing and distribution unit, downstream auto unit, and public service unit [4-5]. With the unit as level, steel-auto industry chain can more accord with the status of the industry chain, better reflect the development trend of the industry chain, and easier implement application of industrial relations model.

Steel-auto industry chain is the process by surrounding from steel coil production, cutting (namely, rolled plate) and processing (namely, auto parts) to ultimately selling to the auto unit, covering product development, manufacturing, processing, inventory, distribution and services, etc. Therefore, the paper considers that the product resources of steel-auto 
industry chain refer to product-related data, knowledge abstraction and integration in whole life cycle, such as the product development, manufacturing, processing, inventory, distribution and service etc.

\section{Grid-based Product Resource Modeling of steel and automobile industry}

With a wide range of applications and in-depth development of information technology in manufacturing, As the basis for enterprise information system data, Product features and process data and other product information resources has become the key to the efficient operation of business processes[6]. Meanwhile, the business process is a product data resource "manufacturing "process, and ultimately the formation of product information, material and nonmaterial performance [7]. But because lack of resources for the effective integration of product data, Causing large amounts of data resources cannot produce the necessary value, which seriously affect the efficiency of business processes and performance levels.

\section{A. Full-information model of product resources}

The purpose of product resource modeling resources into the product chain collaboration throughout the business process in the automobile and steel industry, such as the project collaboration, manufacturing, collaboration, process collaboration, distribution, collaboration, inventory coordination, research and development collaboration and service coordination, etc. to achieve flexible integration of Products and resources with business processes. Combination of iron and steel products, automotive industry chain characteristics, this article feature product information resources into information products, product process resources and product knowledge resources.

1) Information on product features. Product features product information is the basis of information resources and the basic properties of the product and performance information, Such as product name, type, provider, feature information, physical properties, and mechanical properties.

2) Product process resources. Product process resources [8] refers to the different stages of product life cycle of products and product related processes, resources, such as product information in Production processes, resource information and related manufacturing unit manufacturing resources to provide summary information. For example, Automobile industry chain with a steel production process, describes the production process resources, as shown in Fig. 3.

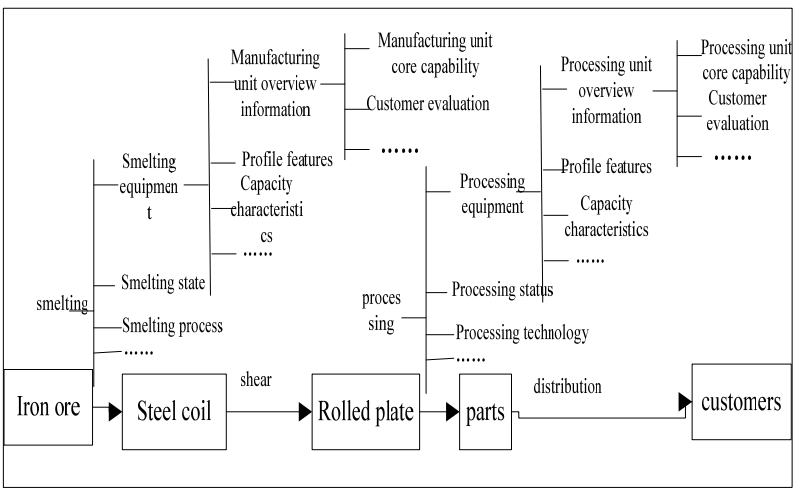

Fig. 3 The product process resources of steel-car industry chain

3) Product knowledge resources. Product knowledge resources is covered by product development, manufacturing, processing, inventory, distribution, and service knowledge and resources in the product during the formation of norms and procedures of operation, Including customer requirements into production planning knowledge, research experience, knowledge of product configuration, manufacturing / process knowledge, processing programs, knowledge of distribution logistics and service knowledge.

Full-information model of Product Resources shown in Fig. 4

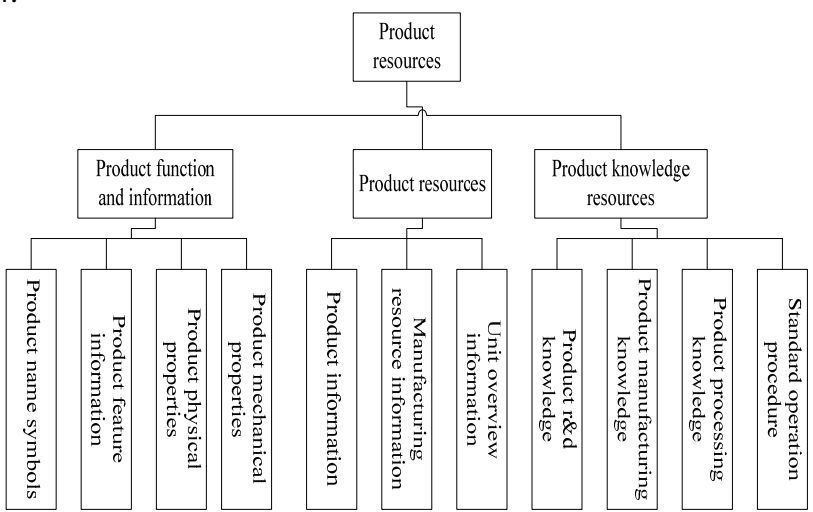

Fig. 4 The all-information model of product resources

\section{B. The formalism description of product resources}

1) The product resources model

Product resources description is an important link in the process such as resource sharing, resource discovery and resource calls, it makes the link of different units' application.

According to the all-information model, expressed the product resources attributes as a triad, $\mathrm{PRA}=\langle\mathrm{ID}, \mathrm{SD}, \mathrm{SB}\rangle$, ID is the product resources mark, which is for the only mark resources; SD (Service Demand) is the Demand information of product resources, which is the basis of resource selection; SB (Service Behavior) is the Behavior information of product resources, which describes the process information of resources [9]. 
ID is the only logo information of product resources; it must distribute by the uniform industry classification, and try to make similar type resources have the adjacent service type coding. ID is mainly corresponding to resource identifier, resources name, type, providers, performance, etc, namely ID: $=\{$ ID, name, type, geo-feature, mecproperty, phyproperty.

SD expressed the demand information of the product resources. In order to realize the different business functions, it including the new product market demand information, plan demand information, technology demand information, manufactures demand information, sales demand information and service demand information, etc. Demand information SD is the basis for users to accurately search appropriate resources according to the business needs.

SB is closely related to the resource identifier and demand information, which expressed the service process of the product resources. It includes all the process, methods, configuration, and events, which is mainly planned according to the product resources demand information.

2) The WSDL unified description of product resources

WSDL [10] is the standard XML format to describe Web services. Use this format, data is unique identity. According to mark information, demand information and behavior information, users can easily search resources to meet conditions with standard way. Take auto plate for example, its attribute information can be formalizing describing as: ALS $=\{$ BaseInfo, ProcessInfo \}, which is:

a) the formal basic properties description for the BaseInfo Resources :

BaseInfo $=$ \{name, category, id, geoFeature , phyProperty, mecProperty , which respectively means the name, type, only identification, geometric features, physical characteristics, mechanical features of the car board. Among them, each element can have further description. Such as, geometric features geo-feature can be further expressed : geoFeature $=\{$ long, wide, high, alignment-Type, alignmentParameter \}, which respectively means the length, width, height, position type, location parameters. The positioning type can be divided into concentric, parallel, phase, etc. properties.

b) The process properties formal description for the ProcessInfo Resources : ProcessInfo $=$ \{manuProperty, taskProperty $\}$, in which manuProperty Processing properties can be further described as: manuProperty $=$ \{resourceNum, machineBody, machineMethod, delivoryMethod\}, Which respectively means resources quantity, processing subject, processing methods, distribution method etc.

TaskProperty Task properties can be further described as: taskProperty $=$ \{machineProcess, delivoryProcess $\}$, Which respectively means Processing and distribution process.

\section{4. the actual application of product resources modeling}

The WSDL document format for auto plate content (part):

<wsdl: types >

$<$ xsd: element name="IDInfo">

$<$ ComplexType $>$

$<$ Sequence $>$ string"/>

$$
\text { <xsd: element name="id"type="xsd: }
$$

$</$ xsd: element $>$

$</$ sequence $>$

$<$ complextype $>$

$</$ xsd: element $>$

$<$ xsd: element name="SDInfo"type="xsd: string">

$<$ Complextype $>$

$<$ Sequence $>$

$<$ sequence $>$

$<$ complextype $>$

$</$ xsd: element $>$

Take the operation getmachineStatus ()for example, it means to get the car plate processing state operation, defined by the interfaces, messages, operations, binding and service elements, the WSDL file format (part):

<wsdl: types> // message data types

$<$ xsd: element name="getMachineStatus" $>$ $<$ ComplexType $>$ $<$ sequence>

$<$ xsd: element name="Zero" type="xsd: int"/>

$<$ sequence $>$

$<$ complexType $>$

$</$ xsd: element $>$

.......

$<$ wsdl: message

<wsdl: binding name="StatusSoapBinding" type="tns:

StatusService"> //SOAP Binding

<soap: binding style="document"

transport="http://schemas.xmlsoap.org/soap/http" />

......

$</$ wsdl: binding $>$

$<$ wsdl: service name="StatusService" $>$ //web Services

$</$ wsdl: service $>$

After a description, registration, etc. products resources become a real grid service, they can share in a collaborative business process.

Take the Vehicle program task for example, products resources' application in the process is show in the Fig. 5: When the user submit the task (XML format) through the client interface to the grid-based collaborative information platform for the steel automotive industry chain, the task Manager use its knowledge to analysis, decomposition and planning, to determine the appropriate coil, and send the request to the Registry service center; Registry service center search coil eligible resources list (resource list state summary of each unit Information); user determine the final unit of partners by the profile information, then you can make the appropriate business process collaboration, such as the project management, production management, etc. 


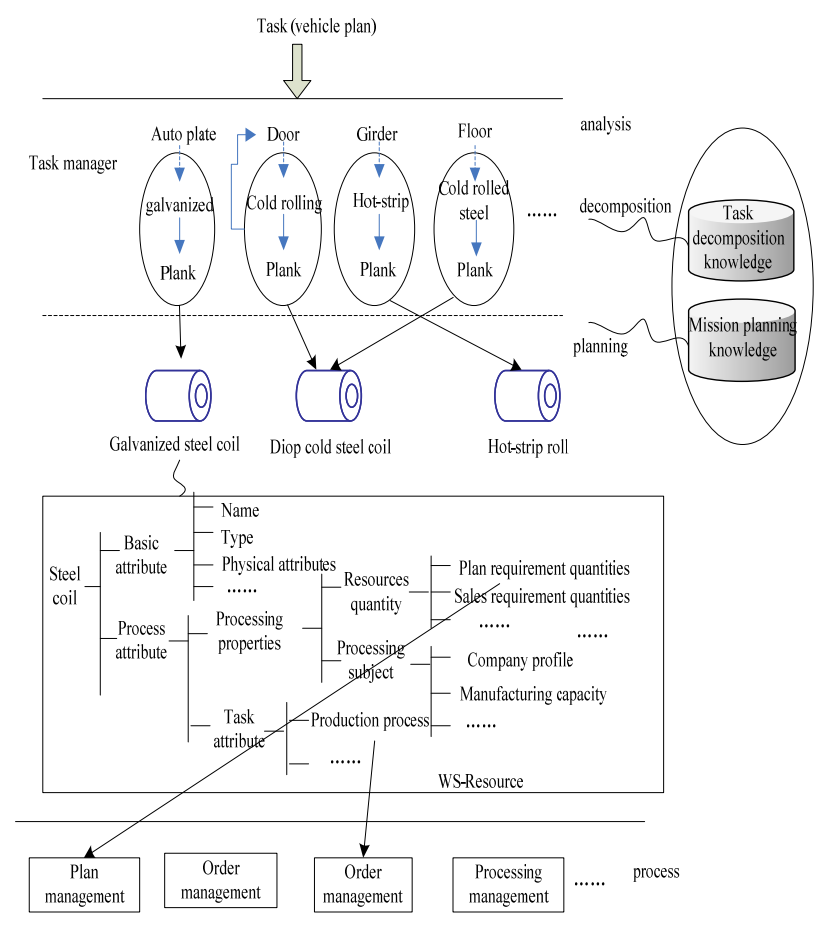

Fig. 5 The application of products resources in the business process

\section{Conclusion}

In this paper, from the perspective of the core business and value added, the steel automobile industry chain is divided into a few units according to its characteristics, such as steel manufacturing unit, trade services unit, processing and distribution unit, downstream car units and public service unit, the product resources is defined, and the full information model, formal description and application of the product resources is studied.

This paper studied the products resources of the steel automobile industry chain, based on this, there is still a lot of work to realize the applications of the steel automotive industry chain collaborative grid information platform, such as the dynamic transformation of product resources, the construction and optimization of the business collaboration model based on products resource, network resource scheduling, grid node construction, operation framework between the nodes, and the operational process research and performance evaluation studies of the whole information platform, etc.

\section{Acknowledgment}

This research work is supported by Scientific Research Plan Projects of Shanghai Science and Technology committee (Research on Industry Chain Collaborative Model Under Ecommerce and Research on Supply Chain Collaborative Supporting Technology for Steel Industry).The authors would like to thank East Steel E-Commerce Co, Ltd. for their funding the work published in this paper.

\section{References}

[1] Ian Foster. "What is the Grid? A Three-point Checklist,” J. Grid Today Daily News and Information for Global Grid Community, vol. 1, no. 6, pp. 10-12, 2002.

[2] Chong-ben Huang and Song-jie Gong, "An Ontology-Based and AgentBased Grid Service Discovery Mechanism,” J. Computer Engineering \& Science, vol. 8, no. 30, pp. 108-110, 2002.

[3] Chen St. "Research in the Supply Chain Management Information System Based on Grid Theory,” D. Shanghai: Tongji University,2008.

[4] Feng Xiong, Zong-hui Xiong and Li Zhen, "Research and Application of Production Resource Collaboration Platform under Manufacturing Grid,”J. Manufacture Information Engineering of China, no. 7, pp. 22-25, 2010

[5] Li Zhen. "Research on Collaboration Information Platform in the Industry Chain of Steel-Auto,” D. Shanghai: Shang University,2010.

[6] Kang Lu, Lai and etc. "The manufacturing data structure in ERP," J. CAD/CAM and Manufacturing Information, no. 4, pp. 16-20, 2004.

[7] C. Passow, M. Pettinatoj and etc. "Break Through factory productivity using e-manufacturing,” J. Solid State Technology, vol. 46, no. 9, pp. 4246, 2003.

[8] Yan-chao Yin and Lin-fu Sun. "Product resource whole performance model based on extension meta-modeling architecture," J. Computer Integrated Manufacturing Systems, vol. 5, no. 15, pp. 905-915, 2009.

[9] Li-Lan Liu, Hongxia Cai and Tao Yu. "Manufacturing grid based on principles and techniques,” ISBN978-7-8118-221-7/TH009, Shanghai University Press.

[10]Wei Sun, Chang-ming Ren and Jiang Zhu. "Grid Resource Framework based in WEB SERVICE -WSRF,” J. Microprocessors, vol. 26, no. 1, pp. 14-15, 2005. 